\title{
Evolution of CT findings in patients with mild COVID-19 pneumonia
}

\section{Ting Liang ${ }^{1} \cdot$ Zhe Liu $^{1} \cdot$ Carol C. Wu ${ }^{2} \cdot$ Chao Jin ${ }^{1} \cdot$ Huifang Zhao ${ }^{1} \cdot$ Yan Wang ${ }^{1} \cdot$ Zekun Wang $^{3} \cdot$ Fen Li ${ }^{3} \cdot$ Jie Zhou $^{4}$.

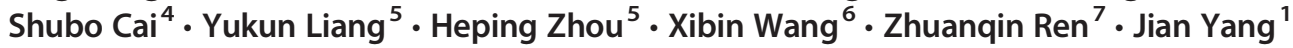

Received: 19 February 2020 / Revised: 14 March 2020 / Accepted: 19 March 2020 / Published online: 15 April 2020

(C) European Society of Radiology 2020

\begin{abstract}
Objectives To delineate the evolution of CT findings in patients with mild COVID-19 pneumonia.

Methods CT images and medical records of 88 patients with confirmed mild COVID-19 pneumonia, a baseline CT, and at least one follow-up CT were retrospectively reviewed. CT features including lobar distribution and presence of ground glass opacities (GGO), consolidation, and linear opacities were analyzed on per-patient basis during each of five time intervals spanning the 3 weeks after disease onset. Total severity scores were calculated.

Results Of patients, $85.2 \%$ had travel history to Wuhan or known contact with infected individuals. The most common symptoms were fever $(84.1 \%)$ and cough $(56.8 \%)$. The baseline CT was obtained on average 5 days from symptom onset. Four patients $(4.5 \%)$ had negative initial CT. Significant differences were found among the time intervals in the proportion of pulmonary lesions that are (1) pure GGO, (2) mixed attenuation, (3) mixed attenuation with linear opacities, (4) consolidation with linear opacities, and (5) pure consolidation. The majority of patients had involvement of $\geq 3$ lobes. Bilateral involvement was more prevalent than unilateral involvement. The proportions of patients observed to have pure GGO or GGO and consolidation decreased over time while the proportion of patients with GGO and linear opacities increased. Total severity score showed an increasing trend in the first 2 weeks.

Conclusions While bilateral GGO are predominant features, CT findings changed during different time intervals in the 3 weeks after symptom onset in patients with COVID-19.

\section{Key Points}

- Four of 88 (4.5\%) patients with COVID-19 had negative initial CT.

- Majority of COVID-19 patients had abnormal CT findings in $\geq 3$ lobes.

- A proportion of patients with pure ground glass opacities decreased over the 3 weeks after symptom onset.
\end{abstract}

Keywords Tomography $\cdot$ Coronavirus $\cdot$ Pneumonia

\section{Abbreviations}

GGO Ground glass opacities

MERS Middle East respiratory syndrome

Ting Liang and Zhe Liu contributed equally to this work.

Carol C. Wu

carolcwu@gmail.com

Jian Yang

yj1118@mail.xjtu.edu.cn

1 Department of Radiology, The First Affiliated Hospital of Xi'an Jiaotong University, Xi'an 710061, People's Republic of China

2 Department of Diagnostic Radiology, University of Texas M.D. Anderson Cancer Center, Houston, TX 77030, USA

3 Department of Radiology, the Eighth Hospital of Xi'an, Xi'an 710061, People's Republic of China
SARS Severe acute respiratory syndrome

COVID-19 Coronavirus disease 19
4 Department of Radiology, Xi'an Chest Hospital, Xi'an 710100, People's Republic of China

5 Department of Radiology, Ankang Center Hospital, Ankang 725000, People's Republic of China

6 Department of Radiology, Hanzhong Center Hospital, Hanzhong 723000, People's Republic of China

7 Department of Radiology, Baoji Center Hospital, Baoji 721008, People's Republic of China 


\section{Introduction}

After multiple cases of pneumonia of unknown etiology were reported in Wuhan, China, in December of 2019, a novel coronavirus was identified in January 2020 and officially named coronavirus disease 19 (COVID-19) by the World Health Organization (WHO) on February 11. Coronavirus infections in human mostly cause mild respiratory tract infections; however, different strains of coronaviruses were also responsible for severe acute respiratory syndrome (SARS) and Middle East respiratory syndrome (MERS) [1-3].

Many of the early cases occurred in those with exposure to the Huanan Seafood Market in Wuhan [2], but the infection has since spread, particularly as many residents left Wuhan for the extended holidays around the lunar new year prior to government shutdown of the city on January 23 . The outbreak of COVID-19 has been declared a global health emergency by the WHO on January 30. As of February 17, 2020, confirmed cases of COVID-19 has been reported in 29 countries and territories; the majority of reported cases remain within China with 803 out of 71,356 cases outside of mainland China, initially in patients who have traveled to China and their close contacts [4]. However, the development of a pandemic seems unavoidable given the natural evolution of the disease, making it a worldwide health problem.

Common presenting symptoms for patients with confirmed infection include fever, cough, and myalgia and fatigue [1-3]. Typical laboratory findings on admission include normal leucocytes in majority of patients and decreased lymphocytes in 35\% of patients [2]. According to published reports, common CT findings consist of bilateral patchy ground glass opacities with peripheral predominance $[5,6]$. As the disease progresses, consolidation becomes more common [6]. Acute respiratory distress syndromes (ARDS) develop in up to $30 \%$ of patients [1]. Intrathoracic lymphadenopathy and pleural effusions are uncommon findings $[2,5]$. Chest radiographic (CXR) findings include bilateral patchy opacities $[3,7]$ though CXRs are considered unreliable in detecting early phase of the pneumonia [8]. Negative chest CTs have been reported in $14 \%$ of patients in one study [5]. Only a small number of cases with follow-up imaging have been reported thus far $[6,9]$. Therefore, we evaluated serial CT pneumonia in patients with mild COVID-19 outside of Wuhan to elucidate the evolution of CT features with correlation to clinical findings.

\section{Materials and methods}

\section{Patients}

A retrospective review of patient data and imaging studies was approved by the institutional review board. No patient consent was required for this HIPAA-compliant study.
Between January 22 and February 8, 2020, 88 patients with COVID-19 were identified from six hospitals in Shaanxi Province in China, which is approximately $800 \mathrm{~km}$ northwest of Wuhan. The inclusion criteria were as follows: (1) patients with COVID-19 confirmed by at least one positive nucleic acid test performed at the Center for Disease Control and Prevention in Shaanxi Province in China and (2) patients who underwent baseline and at least one follow-up chest CT. According to the WHO, patients are divided into six types: uncomplicated illness, mild pneumonia, severe pneumonia, acute respiratory distress syndrome, sepsis, and septic shock [10]. All the patients were treated based on a standard diagnosis and treatment criteria for COVID-19 which includes initiation of antivirals, interferon, Chinese herbal medications, supplemental oxygen as needed, and hospitalization.

\section{Image acquisition}

CT scans of the chest were acquired on 16- to 64-multidector CT scanners (Philips Brilliant 16, Philips Healthcare; GE LightSpeed 16, GE Healthcare; GE VCT LightSpeed 64, GE Healthcare; Somatom Sensation 64, Siemens Healthcare; Somatom AS, Siemens Healthcare; Somatom Spirit, Siemens Healthcare; GE Optima 680, GE Healthcare). The CT parameters were as follows: $120 \mathrm{kVp}$, current intelligent control (auto $\mathrm{mA}$ ) of 30-300 mA, and slice thickness reconstructions of $0.6-3.0 \mathrm{~mm}$. All CT examinations were performed without intravenous contrast material.

\section{Data collection and evaluation}

The medical records of patients were reviewed to determine the demographic data, medical history, exposure history, clinical symptoms, laboratory findings, and time of disease onset. The date of disease onset was defined as patients' reported date of symptom onset. The time intervals, in days, between each CT scan and disease onset were determined and categorized into five groups (days $0-3$, days $4-7$, days $8-10$, days 11-14, and days 15-21).

CT images were reviewed independently by two thoracic radiologists each with 10 years of experience. Differences were resolved by discussion to reach consensus. Similar to a previously published report [6], CT findings including the presence and distribution of ground glass opacities (GGO), consolidation, linear opacities, discrete pulmonary nodules, pleural effusion, lymphadenopathy, and cavitation were evaluated. Degree of lobar involvement and overall lung total severity score were recorded. Each of the five lung lobes was assessed for degree or area of involvement and assigned a score of 0 for $0 \%$ lobe involvement, 1 for $1-25 \%$ lobe involvement, 2 for $26-50 \%$ lobe involvement, 3 for $51-75 \%$ lobe involvement, or 4 for $76-100 \%$ lobe involvement. An 
overall lung total severity score was reached by summing the five lobe scores (range of possible scores, 0-20) [5].

\section{Statistical analysis}

Continuous variables were represented as means and standard deviations, while categorical variables were expressed as counts and percentages. Differences of CT characteristics, lobar involvement, and overall total severity score among different time intervals were statistically compared by the chisquare test or analysis of variance (ANOVA). Linear regression analysis was further used to characterize the linear evolution trends of the variables that were significant at the above analysis.

Statistical analysis was performed by using $\mathrm{R}$ software (version 3.6.0; http://www.Rproject.org). The packages in $\mathrm{R}$ that were used in this study are "gmodels" packages. All $p$ values were considered statistically significant at $p<0.05$.

\section{Results}

\section{Patient demographics}

Of 88 patients in the study cohort, $51(58.0 \%)$ were male and $37(42.0 \%)$ were female; mean age was 42.7 years (range, 4 82 years). Fifty-three $(60.2 \%)$ patients had 2 CTs, 24 (27.2\%) had 3 CTs, and $11(12.5 \%)$ had 4 or more CTs. The mean time interval between symptom onset and baseline CT was $5.5 \pm$ 3.5 days (range, $1-15$ days). Five (5.7\%) patients in the study cohort were known to have been discharged from the hospital. All of the patients had mild pneumonia based on the WHO definition [10].

The demographics of the study cohort are detailed in Table 1. Patients with exposure history (recent travel to Wuhan or contact with infected patient) accounted for $85.2 \%$ of study cohort. Fever (84.1\%) and cough (56.8\%) were the most common presenting symptoms. Low lymphocyte and white cell counts were observed in $26.4 \%$ and $25.0 \%$ of patients, while high C-reactive protein was observed in $55.0 \%$ of patients.

\section{Evolution of CT findings in patients with COVID-19}

\section{Evolution of CT characteristics of pulmonary lesions}

Figure 1 depicts the evolution of CT features of pulmonary lesions in different time intervals from disease onset. Significant differences were found among the time intervals in the proportion of pulmonary lesions that are (1) pure GGO $\left(\chi^{2}=37.97, p=0.01\right)$, (2) mixed attenuation (GGO and consolidation) $\left(\chi^{2}=12.80, p<0.01\right)$, (3) mixed attenuation with reticular/linear opacities $\left(\chi^{2}=20.57, p<0.01\right)$, and (4) consolidation with reticular/linear opacities $\left(\chi^{2}=32.49\right.$, $p<0.01)$ and pure consolidation $\left(\chi^{2}=10.37, p=0.03\right)$. A significant linear decrease in the proportions of pure GGO lesions $(y=0.55-0.08 x, p<0.01)$ and mixed attenuation lesions $(y=0.31-0.03 x, p=0.01)$ and a significant linear increase in the proportions of lesion with reticular/linear opacities (mixed attenuation + linear opacities $(y=0.08+0.04 x$, $p<0.01)$ and consolidation + linear opacities $(y=-0.03+$ $0.04 x, p<0.01)$ ) were observed over time.

\section{Evolution of lobar distribution in patients with COVID-19}

No statistically significant difference was found in the lobar distribution of pulmonary findings over different time intervals from symptom onset, but some trends were observed. For example, the proportion of patients with fewer than 3 lobes affected decreased in the first 10 days (Fig. 2a). The proportion of patients with three or more affected lobes was higher in all of the time intervals analyzed. Bilateral involvement was more prevalent than unilateral involvement with a trend toward an increase in the proportion of patients with bilateral CT abnormalities in the first 2 weeks after disease onset (Fig. 2b). Lower lobes have higher rates of involvement than the others, but the differences are not statistically significant (Fig. 2c).

\section{Evolution of CT findings in patients with COVID-19}

The initial CTs of 4 out of 88 (4.5\%) patients were negative. Two of these four patients had their baseline CT during days $1-3$, one during days $4-7$, and one during days $8-10$ of disease. Subsequently, CT images of one patient remained negative even during days $8-10$, and other patients developed CT abnormalities.

There were significant differences between the time interval groups after disease onset in proportions of patients with both GGO and consolidation $\left(\chi^{2}=22.2, p<0.01\right)$ and the combination of GGO, consolidation, and linear opacities $\left(\chi^{2}=21.5, p<0.01\right)$ (Table 2$)$. A proportion of patients with GGO and consolidation presented a decreasing trend during the 21 days after disease onset $(y=0.48-0.09 x, p<0.01)$, while a proportion of patient with all three findings including ground glass opacities, consolidation, and linear opacities presented an increasing trend $(y=0.12-0.11 x, p<0.01)$. No significant difference was found in the proportions of patients with other CT findings or total lung severity score.

None of the patients had pleural effusions or intrathoracic lymphadenopathy during the follow-up period. Some of the patients had cavitation on CT, but these are thought to represent pre-existing conditions as none of the patients in the study cohort developed cavitation on follow-up CT.

Figures 3 and 4 show the evolution of CT findings of two patients with COVID-19 pneumonia. 
Table 1 Patient demographics and laboratory test results

\begin{tabular}{|c|c|}
\hline Characteristic & Patient with NCP $(n=88)$ \\
\hline Age (years) ${ }^{\mathrm{a}}$ & $42.7 \pm 14.5$ \\
\hline \multicolumn{2}{|l|}{ Age group (years) } \\
\hline$<15$ & $2(2.3)$ \\
\hline $15-44$ & $48(54.5)$ \\
\hline $45-64$ & $28(31.8)$ \\
\hline$\geq 65$ & $10(11.4)$ \\
\hline Male sex & $51(58.0)$ \\
\hline \multicolumn{2}{|l|}{ Exposure history } \\
\hline Recent travel to Wuhan & $45(51.1)$ \\
\hline Contact with infected patient & $30(34.1)$ \\
\hline Unknown exposure & $13(14.8)$ \\
\hline \multicolumn{2}{|l|}{ Initial symptoms } \\
\hline Fever & $74(84.1)$ \\
\hline Cough & $50(56.8)$ \\
\hline Expectoration & $19(21.6)$ \\
\hline Fatigue & $15(17.0)$ \\
\hline Chest distress and/or shortness of breath & $12(13.6)$ \\
\hline Pharyngalgia & $10(11.4)$ \\
\hline Muscle soreness & $7(8.0)$ \\
\hline Headache & $5(5.7)$ \\
\hline Nausea and/or vomiting & $0(0)$ \\
\hline Diarrhea & $0(0)$ \\
\hline No obvious symptoms & $3(3.4)$ \\
\hline \multicolumn{2}{|l|}{ Laboratory testing at initial hospital visit ${ }^{\mathrm{b}}$} \\
\hline White blood cell count $(-, \uparrow, \downarrow)$ & $62(70.5), 4(4.5), 22(25.0)$ \\
\hline Lymphocyte count $(-, \uparrow, \downarrow)$ & 62 (71.3), 2 (2.3), 23 (26.4) \\
\hline Percent lymphocytes $(-, \uparrow, \downarrow)$ & 55 (62.5), 7 (8.0), $26(29.5)$ \\
\hline Percent neutrophilic granulocyte $(-, \uparrow, \downarrow)$ & 59 (67.1), 17 (19.3), 12 (13.6) \\
\hline Percent monocytes $(-, \uparrow, \downarrow)$ & $59(67.1), 26(29.5), 3(3.4)$ \\
\hline Hemoglobin $(-, \uparrow, \downarrow)$ & 68 (77.3), $8(9.1), 12(13.6)$ \\
\hline $\operatorname{ALT}(-, \uparrow, \downarrow)$ & 70 (79.5), 16 (18.2), 2 (2.3) \\
\hline $\operatorname{AST}(-, \uparrow, \downarrow)$ & $73(83.0), 15(17.0), 0(0)$ \\
\hline Creatine kinase $(-, \uparrow, \downarrow)$ & 73 (84.9), 4 (4.6), 9 (10.5) \\
\hline C-reactive protein $(-, \uparrow, \downarrow)$ & $36(45.0), 44(55.0), 0(0)$ \\
\hline
\end{tabular}

Unless otherwise indicated, data are reported as the number of patients, with percentages in parentheses

$A L T$ alanine aminotransferase, $A S T$ aspartate aminotransferase

${ }^{a}$ Data are reported as the mean \pm standard derivation

${ }^{\mathrm{b}}$ The signs - $\uparrow$, and $\downarrow$ represent within, above, and below normal ranges of laboratory results, respectively. Normal ranges of white blood cell count, lymphocyte count, percent lymphocytes, percent neutrophilic granulocyte, percent monocytes, ALT, AST, creatine kinase, hemoglobin, and C-reactive protein were 3.5-9.5 × 10 $0^{9} \mathrm{~L}, 1.10-3.20 \times 10^{9} / \mathrm{L}, 20-50 \%, 3.0-10.0 \%, 40-75 \%, 115-150 \mathrm{~g} / \mathrm{L}, 7-40 \mathrm{U} / \mathrm{L}, 13-35 \mathrm{U} / \mathrm{L}, 40-200 \mathrm{U} / \mathrm{L}$, and 0-10 mg/L, respectively

\section{Discussion}

Patient demographics in terms of age and gender distributions are similar to other published studies [5, 6]. Clinical symptoms and laboratory findings also match those of other initial reports with smaller cohorts $[5,6]$ with fever and cough in the majority of the patients. The majority patients had normal white blood cell count and lymphocyte counts at presentation though $26.4 \%$ of lymphocyte counts below normal range. Of patients in this study, $85.2 \%$ had traveled from Wuhan or known contacts with infected individuals. Notably, $14.8 \%$ of patients in this study based in Shaanxi did not have known or identifiable exposures. The source of infection may become 

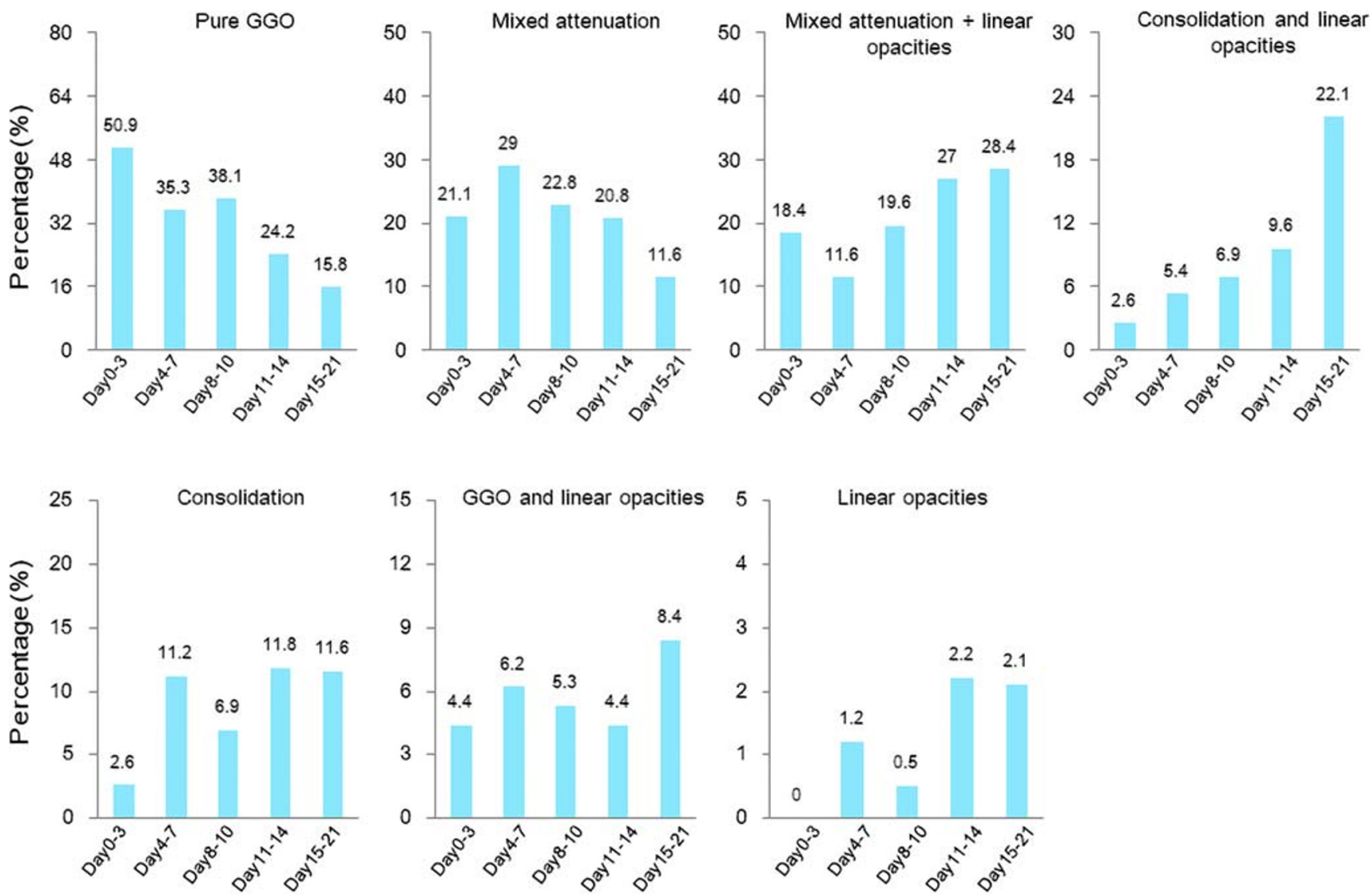

Fig. 1 Evolution of CT characteristics of pulmonary lesions in patients with COVID-19. The time intervals after disease onset (days) were divided into 5 groups, i.e., days 0-3 (lesion number $=114$ ), days 4-7 (lesion number $=241)$, days $8-10($ lesion number $=189)$, days $11-14($ lesion

increasingly difficult to identify if the disease becomes more widespread.

Depending on the severity of clinical symptoms, patients do not always present within the first few days of symptom onset. In our study cohort, the interval between symptom onset and first chest CT ranged from 1 to 15 days. Hence, familiarity with evolution of CT findings is useful to radiologists. Ground glass opacities are the most common CT findings within $0-3$ days of symptom onsets as described in other published reports focused on initial presentations [11]. Over time, GGO remains a common finding and consolidation occurs with higher frequencies than in the early phase of disease, which means the disease is progressing rapidly. It is different from what happened with SARS [12]. Frequency of consolidation decreases 2 weeks after symptom onset. Reticulations and linear opacities, signs of interstitial involvement, and fibrosis become increasing prevalent later in the disease course. The total severity score shows a slight decrease in the third week. Cavitations were present in a small percentage of patients and likely present pre-existing conditions as none of the patients in the study cohort were observed to develop cavitation during the course of COVID-19. Lymphadenopathy and number $=178$ ), and days 15-21 (lesion number $=95)$. GGO, ground glass opacities; mixed attenuation $=$ ground glass opacities + consolidation

pleural effusions were absent on all the CTs analyzed, even on scans obtained 15-21 days after symptom onset. These findings are also similar to other reports [5]. Also similar to other published studies [13], we have observed a lower lobe predominance of pulmonary involvement. Bilateral involvement is found in the majority of patients though a significant number of patients do have unilateral involvement. Awareness of a significant minority of patients with only unilateral findings on CT at various time intervals is important so that radiologists do not exclude the possibility of COVID-19 simply because the findings were unilateral. Finally, in some patients, the CT findings can be minimal or even negative.

In areas outside of China, influenza remains significantly more prevalent at the current time. Common imaging findings of influenza virus-associated pneumonia include GGO, consolidation, and a combination of both. Pleural effusion, if present, is usually minimal, and lymphadenopathy is rare $[14,15]$. Organizing pneumonia has also been reported [16]. There are recent publications that have suggested that CT findings of COVID-19 are similar to the organizing pneumonia (OP) pattern observed from post viral or atypical infections and other etiologies [17]. Despite a study 

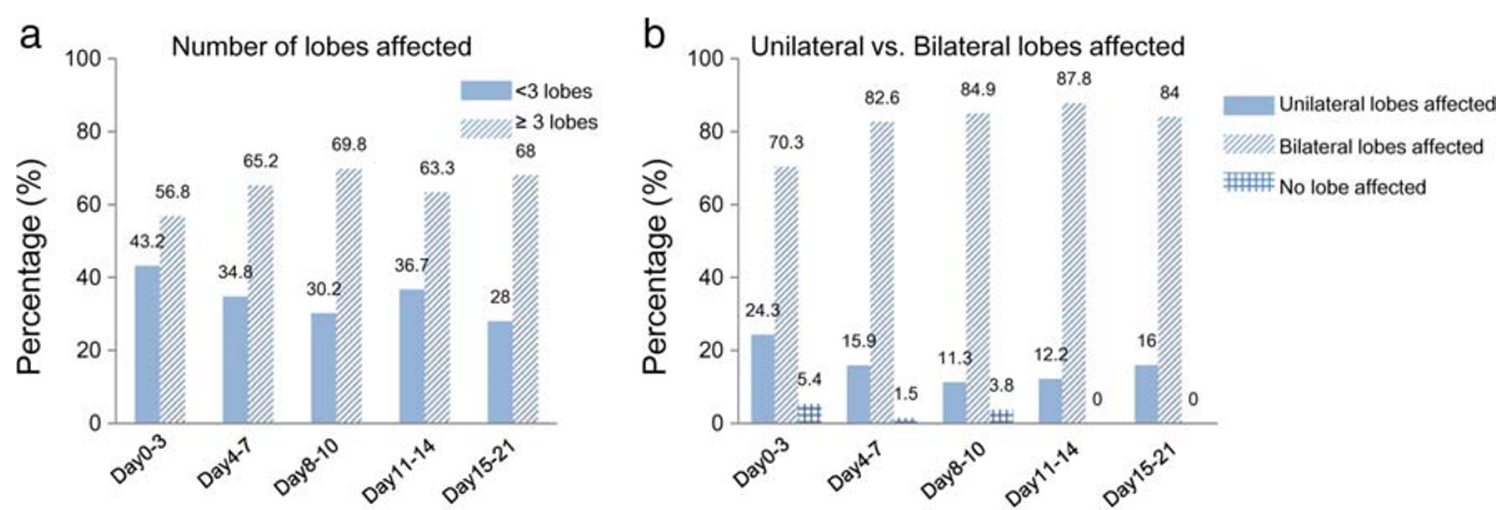

C

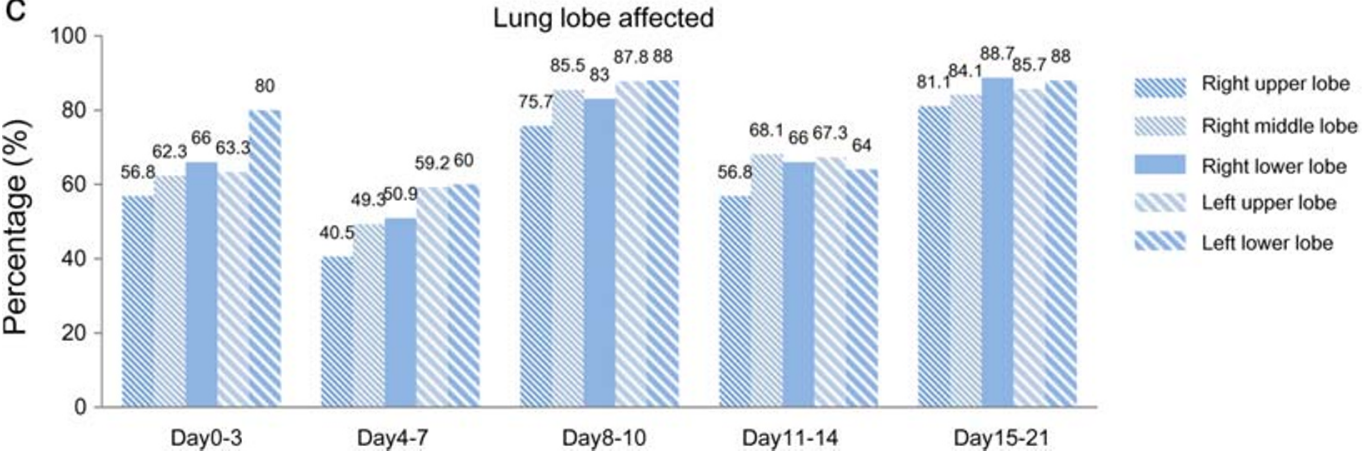

Fig. 2 Evolution of lobar distribution of the (a) number of lobe affected, (b) unilateral and bilateral lobes affected, and (c) lung lobe affected in patients with COVID-19 within 21 days after disease onset. The time after disease onset (day) is categorized as 5 groups, i.e., days 0-3 (patient

number $=37)$, days $4-7$ (patient number $=69)$, days $8-10$ (patient number $=53)$, days $11-14$ (patient number $=49)$, and days 15-21 (patient number $=25$ )

Table 2 Evolution of CT findings and total lung severity scores of patients with COVID-19 within 21 days after disease onset

\begin{tabular}{|c|c|c|c|c|c|c|c|}
\hline \multirow[t]{2}{*}{ Characteristic } & \multicolumn{5}{|c|}{ Time after disease onset } & \multirow[t]{2}{*}{$\chi^{2}$} & \multirow{2}{*}{$\begin{array}{l}p \\
\text { value }\end{array}$} \\
\hline & $\begin{array}{l}\text { Days 0-3 } \\
(n=37)\end{array}$ & $\begin{array}{l}\text { Days 4-7 } \\
(n=69)\end{array}$ & $\begin{array}{l}\text { Days 8-10 } \\
(n=53)\end{array}$ & $\begin{array}{l}\text { Days } 11-14 \\
(n=49)\end{array}$ & $\begin{array}{l}\text { Days 15-21 } \\
(n=25)\end{array}$ & & \\
\hline \multicolumn{8}{|l|}{ Pulmonary opacities } \\
\hline GGO only & $8(21.6)$ & $13(18.8)$ & $7(13.2)$ & $4(8.2)$ & $1(4.0)$ & 6.6 & 0.16 \\
\hline GGO and consolidation & $12(32.4)$ & $26(37.7)$ & $8(15.1)$ & $5(10.1)$ & $1(4.0)$ & 22.2 & $<0.01$ \\
\hline GGO and linear opacities & $2(5.4)$ & $2(2.9)$ & $4(7.5)$ & $2(4.1)$ & $2(8.0)$ & 1.87 & 0.76 \\
\hline Consolidation only & $1(2.8)$ & $6(8.7)$ & $3(5.7)$ & $4(8.2)$ & $1(4.0)$ & 1.98 & 0.74 \\
\hline Consolidation and linear opacities & $0(0)$ & $2(2.9)$ & $4(7.5)$ & $4(8.2)$ & $3(12.0)$ & 6.0 & 0.19 \\
\hline $\begin{array}{l}\text { GGO, consolidation, and linear } \\
\text { opacities }\end{array}$ & $12(32.4)$ & $19(27.5)$ & $25(47.2)$ & $30(61.2)$ & $17(68.0)$ & 21.5 & $<0.01$ \\
\hline Negative CT & $2(5.4)$ & $1(1.5)$ & $2(3.8)$ & $0(0.0)$ & $0(0.0)$ & 4.3 & 0.36 \\
\hline \multicolumn{8}{|l|}{ Other findings } \\
\hline Discrete pulmonary nodules & $4(10.8)$ & $4(5.8)$ & $3(5.7)$ & $1(2.0)$ & $0(0)$ & 4.8 & 0.36 \\
\hline Pleural effusion & $0(0)$ & $0(0)$ & $0(0)$ & $0(0)$ & $0(0)$ & - & - \\
\hline Lymphadenopathy & $0(0)$ & $0(0)$ & $0(0)$ & $0(0)$ & $0(0)$ & - & - \\
\hline Cavitation & $3(8.1)$ & $3(4.3)$ & $1(1.9)$ & $2(4.1)$ & $1(4.0)$ & 2.1 & 0.72 \\
\hline \multicolumn{8}{|l|}{ Total lung severity score } \\
\hline Mean \pm SD & $3.9 \pm 2.7$ & $4.8 \pm 2.9$ & $4.9 \pm 2.8$ & $5.3 \pm 3.3$ & $5.2 \pm 2.8$ & - & 0.29 \\
\hline Median (range) & $4(0-10)$ & $5(0-12)$ & $5(0-11)$ & $5(1-13)$ & $5(1-11)$ & - & - \\
\hline
\end{tabular}

Numbers in parentheses are percentages

$n$ number of patients, $G G O$ ground glass opacities 
Fig. 3 A 54-year-old woman with exposure to a COVID-infected patient presented with fever, cough, and low back pain for 2 days and remains hospitalized. a-c CT shows left lower lobe GGO on day 2 . The total severity score is 1 . $\mathbf{d - f}$ CT on day 7 shows a significant increase in $\mathrm{GGO}$ in both lungs. The severity score of the left lower lobe is 2 . The total severity score is $5 . \mathbf{g}-\mathbf{i}$ CT on day 9 shows bilateral GGO and subpleural consolidation. The severity score of the left lower lobe is 3 . The total severity score is 9 . j-I CT on day 11 shows diffuse GGO and linear opacities (thick arrow). The severity score of the left lower lobe is 4 . The total severity score is 12
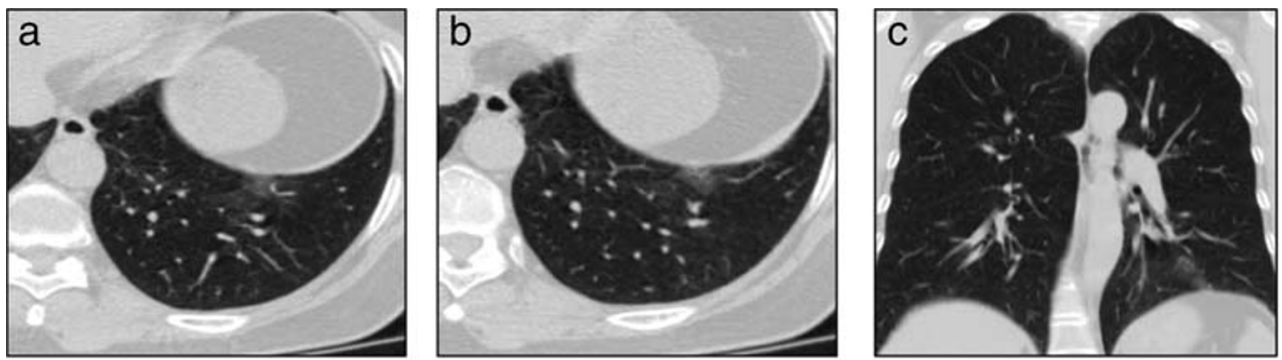

Day 2 Total severity score $=1$
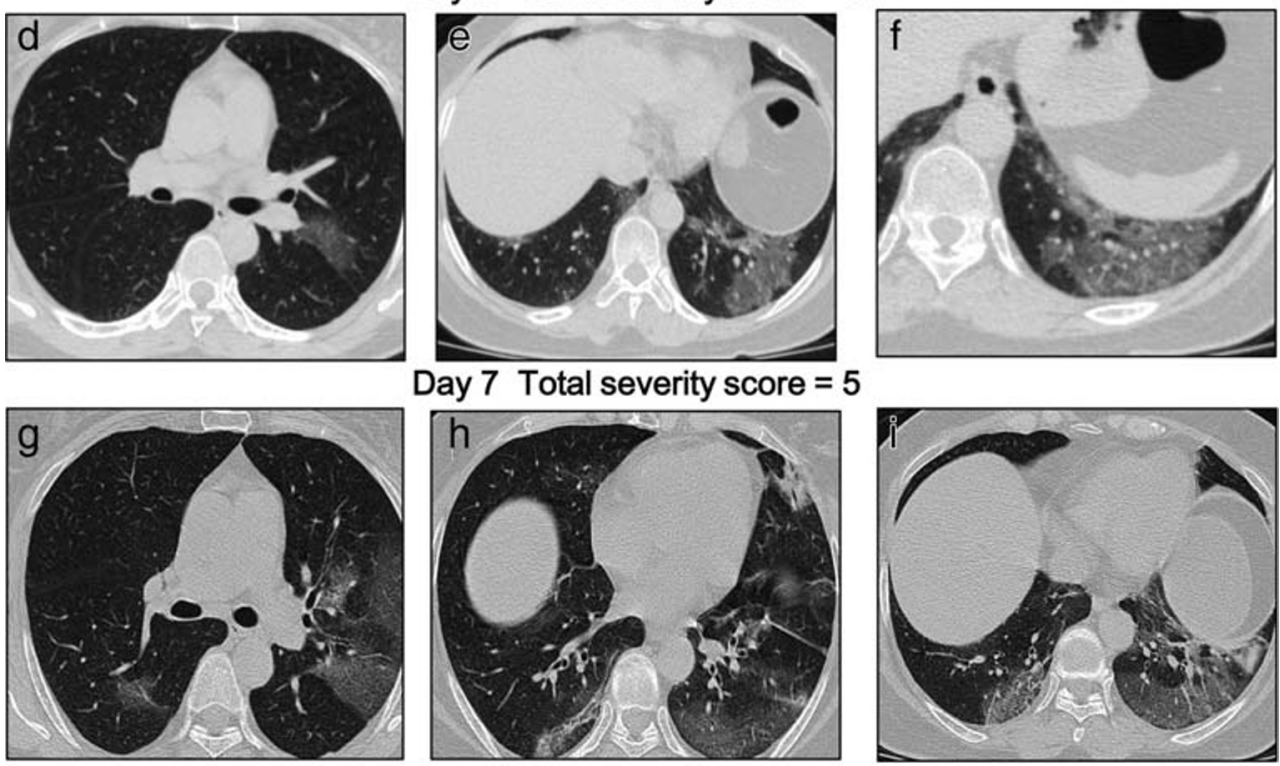

Day 7 Total severity score $=5$

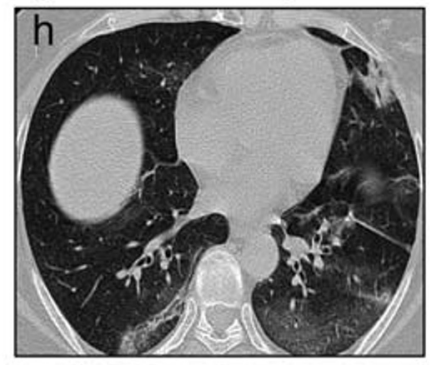

Day 9 Total severity score $=9$
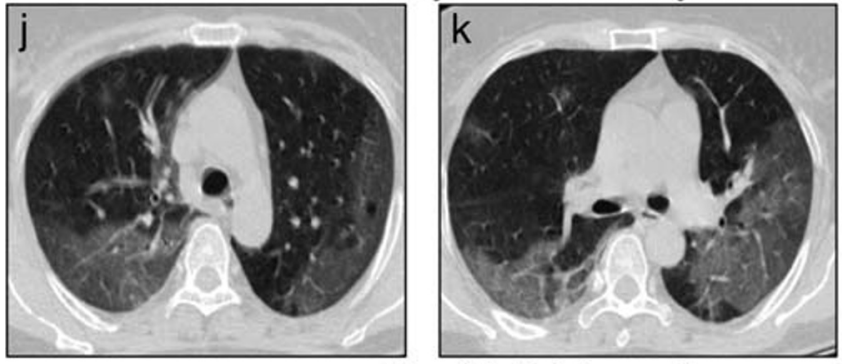

Day 11 Total severity score $=12$
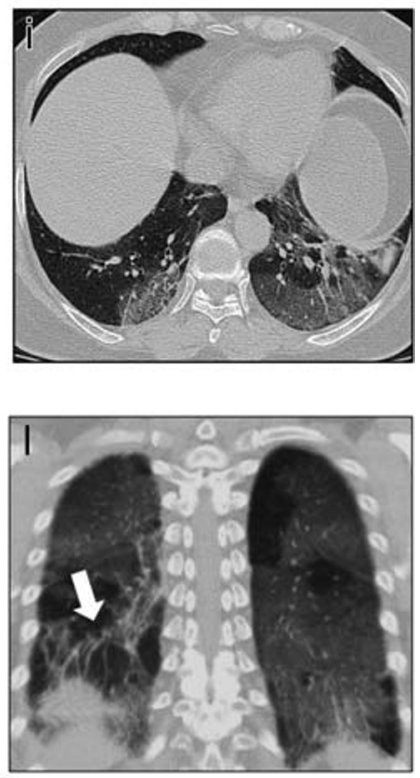

healthcare setting for evaluation. It is possible that there are infected individuals within the population with subclinical or mild clinical symptoms who did not present for care and the findings reported here are skewed toward those who were more symptomatic. Additionally, many of the more critically ill patients were transferred to other hospitals which were not included in this study. Our data were collected from six sites with variable $\mathrm{CT}$ scanning parameters though the data heterogeneity reflects the different practice parameters and settings. The patients did not have CT studies at regular intervals as often is the case in the clinical setting where the timing of imaging is dependent on the clinical course and different for each patient. Finally, even though the study describes evolution of CT findings, the chronic alterations in the pulmonary
Limitations of our study include the retrospective nature of the study where all patients in the cohort presented to 

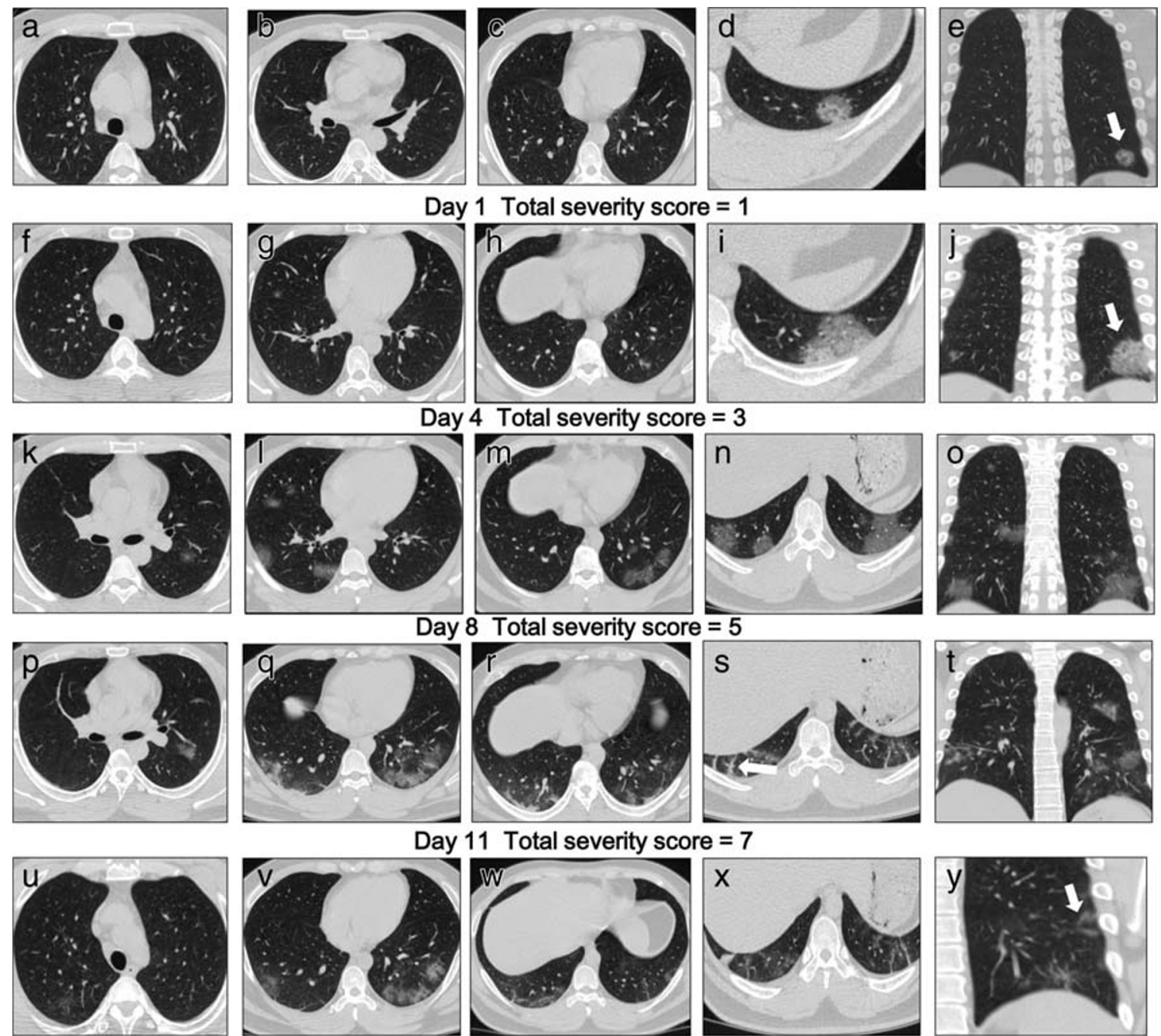

Day 15 Total severity score $=\mathbf{5}$

Fig. 4 A 24-year-old man with exposure to a COVID-infected patient presented with fever and cough for 1 day and remains hospitalized currently. a-e CT scan shows GGO in the subpleural left lower lobe on day 1 (thick arrow). The overall total severity score is $1 . \mathbf{f}-\mathbf{j}$ CT on day 4 shows enlarging GGO in left lower lobes (thick arrow). The overall total severity score is 3 . $\mathbf{k}-\mathbf{0}$ CT on day 8 shows increased GGO in bilateral lower lobes. The total severity score was 5 . p-t CT obtained on day 11 shows bilateral ground glass opacities and linear opacities (thick arrow). The overall total severity score was 7. u-y CT obtained on day 15 shows improvement of bilateral lower lobe GGO and atoll sign (thick arrow). The overall total severity score is 5 parenchyma remain to be determined. A longer follow-up would be needed.

In conclusion, CT findings of patients with mild COVID19 outside of Wuhan include predominance of GGO in the early phase with an increase in the frequency of consolidation and linear opacities over the 3 weeks post onset of symptoms. A small percentage of patients can have no or minimal CT abnormalities. Given that laboratory tests for the novel coronavirus can be falsely negative, radiologists play a key role in identifying suspicious CT findings based on time interval from the onset of symptoms and guide further evaluation and management of patients.
Funding information This study has received funding by the National Key Research and Development Program of China (2016YFC0100300); the National Natural Science Foundation of China (Nos. 81171317, 81471631, 81771810, and 51706178), the 2011 New Century Excellent Talent Support Plan of the Ministry of Education, China (NCET-110438); the China Postdoctoral Science Foundation (No. 2017M613145); and the Clinical Research Award of the First Affiliated Hospital of Xi'an Jiaotong University (No. XJTU1AF-CRF-2015-004).

\section{Compliance with ethical standards}

Guarantor The scientific guarantor of this publication is Jian Yang, Department of Radiology, the First Affiliated Hospital of Xi'an Jiaotong University. 
Conflict of interest The authors declare that they have no conflict of interest.

Statistics and biometry No complex statistical methods were necessary for this paper.

Informed consent Written informed consent was obtained from all subjects (patients) in this study.

Ethical approval This study was approved by the Ethics Committee of the First Affiliated Hospital of Xi'an Jiaotong University. Institutional review board approval was obtained.

\section{Methodology}

- retrospective

- diagnostic or prognostic study

- performed at six institutions

\section{References}

1. Huang C, Wang Y, Li X et al (2020) Clinical features of patients infected with 2019 novel coronavirus in Wuhan, China. Lancet 395: 497-506

2. Chen N, Zhou M, Dong X et al (2020) Epidemiological and clinical characteristics of 99 cases of 2019 novel coronavirus pneumonia in Wuhan, China: a descriptive study. Lancet 395:507-513

3. Zhu N, Zhang D, Wang $\mathrm{W}$ et al (2020) A novel coronavirus from patients with pneumonia in China, 2019. N Engl J Med 382:727733

4. Coronavirus toll update: cases and deaths by country and territory. https://www.worldometers.info/coronavirus/. Accessed 12 Feb 2020

5. Chung M, Bernheim A, Mei X et al (2020) CT imaging features of 2019 novel coronavirus (2019-nCoV). Radiology 295:202-207

6. Song F, Shi N, Shan F et al (2020) Emerging coronavirus 2019nCoV pneumonia. Radiology 295:210-217

7. Phan LT, Nguyen TV, Luong QC et al (2020) Importation and human-to-human transmission of a novel coronavirus in Vietnam. N Engl J Med 382:872-874

8. Holshue ML, DeBolt C, Lindquist S et al (2020) First case of 2019 novel coronavirus in the United States. N Engl J Med 382:929-936
9. Pan F, Ye T, Sun P et al (2020) Time course of lung changes on chest CT during recovery from 2019 novel coronavirus (COVID19) pneumonia. Radiology. https://doi.org/10.1148/radiol. 2020200370

10. World Health Organization (2020) Clinical management of severe acute respiratory infection when COVID-19 is suspected. https:// www.who.int/publications-detail/clinical-management-of-severeacute-respiratory-infection-when-novel-coronavirus-(ncov)infection-is-suspected. Accessed 12 Jan 2020

11. Pan $\mathrm{Y}$, Guan $\mathrm{H}$, Zhou S et al (2020) Initial CT findings and temporal changes in patients with the novel coronavirus pneumonia (2019nCoV): a study of 63 patients in Wuhan, China. Eur Radiol. https:// doi.org/10.1007/s00330-020-06731-x

12. Hui JY, Cho DH, Yang MK et al (2003) Severe acute respiratory syndrome: spectrum of high-resolution CT findings and temporal progression of the disease. AJR Am J Roentgenol 181:1525-1538

13. Fang Y, Zhang H, Xu Y, Xie J, Pang P, Ji W (2020) CT manifestations of two cases of 2019 novel coronavirus (2019-nCoV) pneumonia. Radiology 295:208-209

14. Amorim VB, Rodrigues RS, Barreto MM, Zanetti G, Hochhegger B, Marchiori E (2013) Influenza A (H1N1) pneumonia: HRCT findings. J Bras Pneumol 39:323-329

15. Koo HJ, Lim S, Choe J, Choi SH, Sung H, Do KH (2018) Radiographic and CT features of viral pneumonia. Radiographics 38:719-739

16. Fujita J, Bandoh S, Yamaguchi M, Higa F, Tateyama M (2007) Chest CT findings of influenza virus-associated pneumonia in 12 adult patients. Influenza Other Respir Viruses 1:183-187

17. Kanne JP, Little BP, Chung JH, Elicker BM, Ketai LH (2020) Essentials for radiologists on COVID-19: an update - radiology scientific expert panel. Radiology. https://doi.org/10.1148/radiol. 2020200527

18. Bai HX, Hsieh B, Xiong Z et al (2020) Performance of radiologists in differentiating COVID-19 from viral pneumonia on chest CT. Radiology. https://doi.org/10.1148/radiol.2020200823

19. Kanne JP (2020) Chest CT findings in 2019 novel coronavirus (2019-nCoV) infections from Wuhan, China: key points for the radiologist. Radiology 295:16-17

20. Xie X, Zhong Z, Zhao W, Zheng C, Wang F, Liu J (2020) Chest CT for typical 2019-nCoV pneumonia: relationship to negative RTPCR testing. Radiology. https://doi.org/10.1148/radiol.2020200343

Publisher's note Springer Nature remains neutral with regard to jurisdictional claims in published maps and institutional affiliations. 